\title{
Microcontroller based elevator conservation system to reduce energy consumption
}

\author{
Mahnaz Hasan Qabazard ${ }^{1}$, Ali Sobhy Mohamdein Roza ${ }^{2}$ \\ ${ }^{1,2}$ (Electrical Engineering Department, Kuwait University, Kuwait)
}

\begin{abstract}
This paper present a microcontroller based elevator energy conservation system to reduce the energy consumption of a traction elevator and enhance the performance of the elevator. To control the energy conservation of the elevator, here two controllers have been used namely sensor board controller and motor control board. Sensor board controller monitors the load condition and motor control board control the speed, current and voltage of the motor as well as weight of the motor shaft from the sensor board controller. As microcontroller is very micro in size and integrated in a single chip, this controller can be used efficiently to control the elevator conservation system by minimizing the energy consumption. The designed model demonstrates that by controlling the elevator with this controller energy consumption and travelling time of the elevator are reduced compared with the elevator system which is controlled by conventional controller.
\end{abstract}

Keywords: Microcontroller, Traction elevator, Conservation, Energy consumption.

\section{INTRODUCTION}

In today's society, technology is growing at an exponential rate, and more people every day are plugging in gadgets siphoning the planets limited resources [1,2]. The population of the world is nearing 7 billion people and by 2050 the U.S. Census Bureau estimates that number will increase to 9 billion. The demand for fossil fuels and building materials for nuclear power plants is exceeding the supply. These days, it is not uncommon to see stores lining their shelves with "green" items such as clothing and cleaning products. The new "Green Energy" movement has changed the way most engineers approach the problem of powering new technologies they are developing. However, society may have overlooked an important resource. Before the development of semiconductor parts, many technologies were put in place when power was not an issue. This was a time in which the supply of power was greater than the demand. Also engineers were more concerned with functionality of their devices and systems than the power consumption, due to the infancy of most electronics. A good example of such a system would be the traction elevator. This system was created to provide a vertical mode of transportation to buildings that exceeded more than 4 floors. They used a counter balance system and an AC Induction motor controlled by circuitry and buttons. This system has many areas for improvement but the most significant is the AC Induction motor's low power factor. The motor is used at a constant speed because the technology for variable speed using semiconductor parts was not invented at that time. The circumstances of our times have given rise to a need for optimization, and there are many resources hidden in systems that are in place and used every day. So, controlling the AC motor of the elevator system is very important now-a-days to minimize the energy consumption which in turns save the generated energy. Considering this discussion, this paper aims to design a low cost energy conservation system by controlling the motor of the traditional traction elevator system with microcontroller to minimize the energy consumption and increase the enhancement of the performance of it.

An elevator system is a vertical transport vehicle that efficiently moves people or goods between floors of a building. They are generally powered by electric motors. The most popular elevators are the traction elevators which are lifted by ropes and pass over a wheel attached to an electric motor above the elevator shaft. In the traction elevator, the car is raised and lowered by transaction with steel rope.

There are many designs of elevators in the world but the most common is the traction elevator. These types of elevators are designed to use an AC Induction motor as a focal point to counterbalance the weight of a passenger cart. The cart is balanced by a counter weight that weighs the same as the cart itself and 50 percent of the cart's maximum load. Because of this balance, the torque load on the motor itself is caused when a load is added to the cart. As the motor turns, the elevator cart uses the motor force as well as the counterbalance to reach its destination. There are different solutions to enhance the performances of the elevator system. From the different solutions, as mentioned earlier, this paper aims to develop a strategy to minimize the energy consumption and enhance the performance of the elevator system. The focus on this project is the AC Induction motor. These motors usually have a low power factor because of their inefficiency due to the load. The reason for this pertains to the way an AC Induction motor works. The motor charges a stator, or stationary part of the motor and through electromagnetic induction transfers its power to a rotor, or the rotating part of the motor. The speed of the rotor will match the speed of the magnetic field in the stator. Overcoming the initial turn is where 
the motor loses most of its power factor; a varying load also makes the motor wasteful at lower loads. The first AC Induction motor was invented in 1883 and was used mainly in constant speed cases including in traction elevators for many of the high rise buildings used today. In the late 1960's the first semi-conductor transistor was invented and AC Induction motors were able to be used in variable speed applications.

Now that technology has developed and is now moving towards a new green energy era, one can reevaluate traction elevator system [2]. This concept focuses on to accomplish is to vary the speed of the AC Induction motor in order to keep the efficiency of the motor as high as possible. By sensing the load added to the cart, one could transmit that data to a motor controller and use the information along with specifications of the motor to set an appropriate speed. This system has the benefit of less energy consumption throughout the process and a dramatic decrease in travel time. The idea is for this system to be able to integrate into old elevator systems as well as adapt to newer elevator systems.

As mentioned in the background, green energy is the only solution to meet the increasing population demand and minimize the dependency of the conventional sources as well as to mitigate the adverse effect from these sources. Another way among the many possible ways is, to reduce the dependency of the energy by reducing the daily consumption of the energy. When it comes to the field of energy conservation, many firms are specializing in reducing energy and power usage within buildings to restrain expenses. One of the components at the center of attention to save this energy and power are elevators. The most often used design of these units are AC induction motor controlled traction based elevators. This system lowers or raises its load at a constant speed. It is believed that if someone was to alter the speed of the motor based on the load within the elevator, they could potentially save energy usage. So, it is very important to design the controller of the motor to reduce the energy conservation by enhancing the performance of the elevator.

Optimization of power usage in elevator operation has become a major focus for energy conservation. Some study shows that, there are lots of elevators; none of them are fully optimized for energy consumption in Malaysia. Current common elevator controls are achieved through constant speed operation. An optimization process using variable speed control based on elevator load has theoretically shown to reduce energy consumption by up to 11 percent while reducing overall transport times by 20 percent. This paper focuses to implement an Elevator Conservation Controller (ECC) to optimize the energy consumption and enhance the power of elevator by using microcontroller as a controlling device. ECC will involve a load based control algorithm that will verify the previously mentioned theoretical models for traction style elevators.

This work has implemented an idea that someone could realistically decrease the energy consumption of elevators as well as the overall travel time for various loads. It is necessary to maintain some procedures to achieve this goal like to detect the load within the mock-cart with a tension sensor. Also, controller design is very much important to control the motor as here, for this project microcontroller has been used as a controller. This motor controller will control both the motion of the motor as well as collect various data. Upon completion of two runs, one of single speed and one of variable speed, the system will be able to give data about both the energy usage and travel times. With this data, one can verify if the real life measurement conforms to the simulated results or not. It can be verified that, the amount of energy conserved will save expenditure for the owners and/or users of the elevators as well as grant them the benefit of a reduced travel time. Owners and operators could easily implement this system to nearly all existing and future elevators.

\section{AdVANTAGES OF MicROCONTROLLER}

Microcontroller's use increased rapidly. Now these are used in almost every electronic equipment like Washing Machines, Mobile Phones and Microwave Oven. Following are the most important facts about Microcontrollers, which causes rapid growth of their use [3-5]:

- $\quad$ Microcontrollers are cheap and very small in size, therefore they can be embedded on any device.

Programming of Microcontrollers is simple to learn. Its not much complicated.

We can use simulators on Computers to see the practical results of our program. Thus we can work on an Embedded project without even buying the required Components and Chips. Thus we can virtually see the working of our project or program. 


\section{BASIC STRUCTURE OF ELEVATOR SYSTEM}

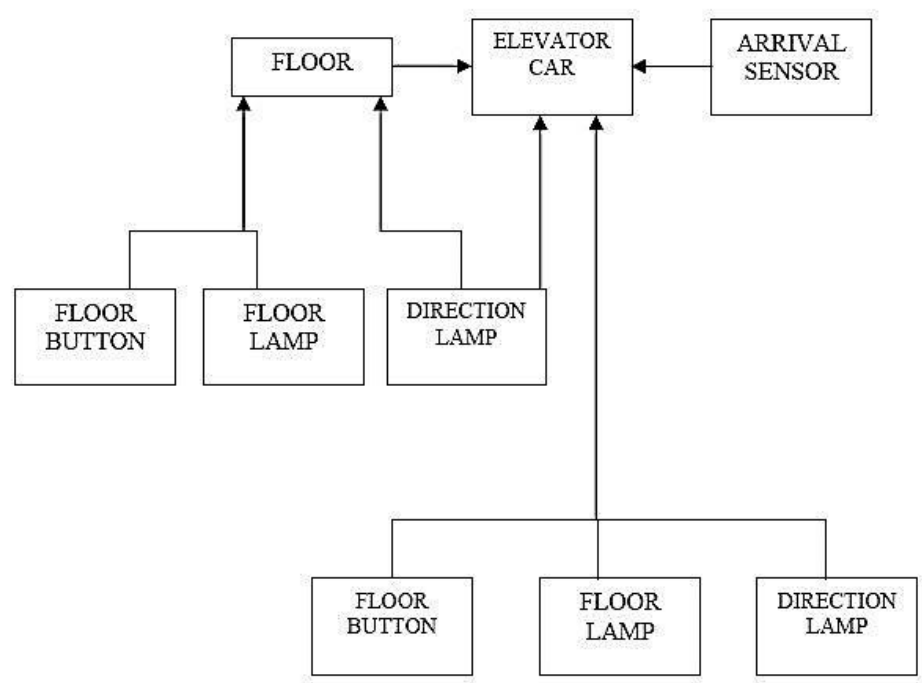

Figure 1. Basic structure of Elevator System

Figure1 shows the elevator system overview [6]. This figure consists of floor where passenger wants to visit. Elevator car moves it either upward or downward direction. The arrival sensor detected the arrival of the elevator to the respective floor. Floor button is used to take the elevator to the respective floor. Floor lamp shows the indication of floor and direction lamp shows the direction of elevator movement, whether it is upward or downward direction. Elevator button is used for moving the elevator car either in upward and downward direction. Based on the elevator switch pressed, the elevator car is moved either in upward and downward direction. D.C. Motor is another important component of elevator system. Based on the switch pressed, the D.C. Motor either moves in forward and reverse direction to move the elevator in either upward or downward direction. Door of the elevator system is one of the important factors of elevator system. When elevator car stops in particular floor, the door of the elevator is opened for passenger to be come out and come in to the elevator car. Arrival sensor is used in every floor, for detecting the elevator car. When a particular car is reached to the particular floor, this arrival sensor detects the elevator car and stops that car.

When User presses an elevator button, the elevator button sensor sends the elevator button request to the system, identifying the destination floor the user wishes to visit. When any new request comes, this new request is added to the list of floors to visit. If the elevator is stationary, the system determines in which direction the system should move in order to service the next request. The system commands the elevator door to close, when user presses the elevator door closed button. When the door has closed, the system commands the motor to start moving the elevator, either in up and down direction, based on switch pressed.

When the elevator moves between floors, the arrival sensor detects that the elevator is approaching a floor and notifies the system to stop the elevator and open the door of the elevator system. Fig. 2 shows the elevator dispatching strategy.

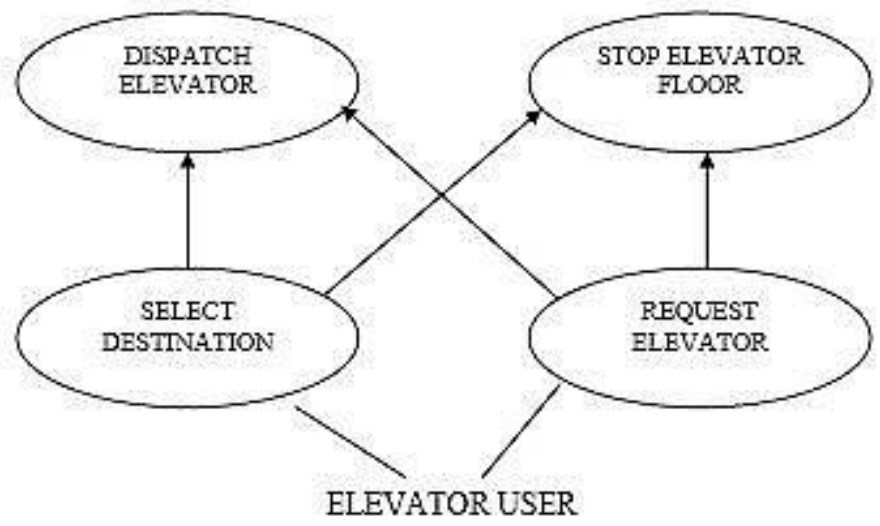

Figure 2. Elevator Dispatching Strategy 


\section{How AN Elevator CONSUMES AND REgENERATES ENERGY}

When an electric motor accelerates or maintains velocity, it consumes energy. But when this same electric motor brakes or decelerates a body in motion, the motor becomes a generator of energy [1]. This energy has traditionally been considered a nuisance, but with the invention of integrated regenerative drives, this "waste" energy is sent back into the electrical grid.

Power is consumed in a traction elevator first, by the gravitational pull on ascending cabs that are heavier than the descending counterweight and second, by the gravitational pull on ascending counterweights when they are heavier than descending elevator cabs. Fig. 3(a) shows the condition when the elevator is in energy consumption state.

Power is generated in a traction elevator first, by the gravitational pull on descending cabs that are heavier than the ascending counterweight and second, by the gravitational pull on descending counterweights when they are heavier than ascending elevator cabs.

In the case of power generation, the mechanical energy of the descending car or counterweight causes the elevator motor to function as a generator (or re-generator) of electricity. The elevator also produces electricity when the motor works as a braking system to decelerate. Conventional elevator systems dissipate this untapped electricity as waste heat, routing it through electrical resistors in the elevator shaft or machine room, using essentially the same principle as an electric toaster. This waste heat is not only inefficient, but can raise the ambient temperatures in elevator machine rooms and often require additional cooling. Fig. 3(b) shows the energy generation mode of a traction elevator.

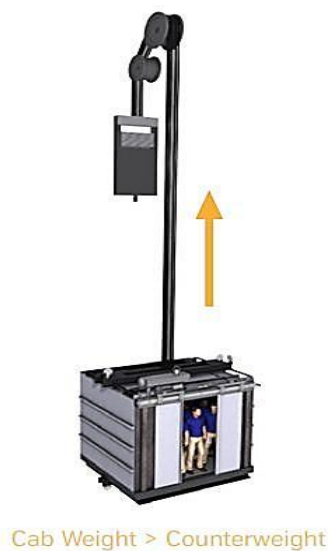

(a)

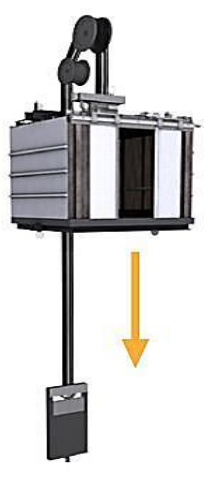

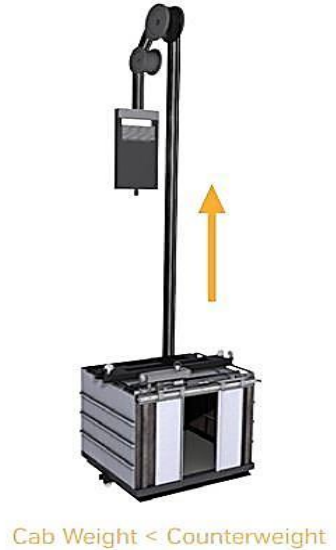

Figure 3. (a) Consuming of Energy, (b) Generation of Energy

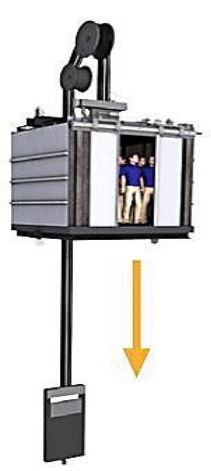

Cab Weight > Counterweight.

(b)

\section{DESIGN OF THE ENERGY EFFICIENT ELEVATOR CONSERVATION SYSTEM}

The design serves an in depth representation of the Elevator Conservation Controller (ECC) which has been built and tested in this paper. The Elevator Conservation Controller can be created to test whether a constant speed or a variable speed elevator controller is more energy efficient based on power consumption and travel time. This paper presents for a small scale mechanical elevator model in order to integrate the control system but this can be enhanced to any other elevator system. The step by step block diagram of the design of energy efficient elevator conservation system is shown in Fig. 4. The elevator car microcontroller board contains the travel pattern and weight which is loaded from the load sensor board. The motor controller board will receive information from the elevator car microcontroller board via XBee. Speed has been calculated in the motor controller board to let the motor know what speed to execute. Power has also been measured in the motor controller board to know the energy consumption. Detailed expectations regarding the Elevator Conservation Controller project are outlined in the following paragraphs.

The complete system is able to simulate three user defined traffic patterns via a hardware switch that has been selected the mode for the system. The Off mode will stop the car and return it to the ground floor; the Constant mode will run with a constant speed regardless of weight; and the Variable mode will run with a varying speed based upon weight. The variable mode scenario will detect the load within the car with a tension sensor. The weight scale will be a 1:40 ratio based on an average calculation derived. A real elevator can hold up to 2,000 pounds, so, for this conceptual proposal it has been scaled down to 50 pounds which established the 1 to 40 ration. The weight will be used to calculate the most efficient speed that the motor needs to run, based on a pre-formulated algorithm created by our customer and the motor specifications. The weight will be transmitted from the elevator car microcontroller board to the motor controller board along with the desired destination. 
Once the information is obtained the motor controller board will execute the appropriate speed, measure the power used, and determine the travel timing.

The system will consist of a car representing the elevator containing a weight sensor board and load weights. A traffic pattern has been created and stored onto the elevator car microcontroller board to have a running pattern that will be sent to the motor controller board to make the elevator car move up and down to the respective floors. The motor controller board controls the motion of the motor as well as collect data. Additionally, a load sensor has been placed in line with the support cable to sense the weight of the load placed on the car during the variable speed scenario. The weight value is sent from the load sensor board to the elevator car microcontroller board through analog input. Once the load value (weight) is on the elevator car microcontroller board it sends that information to the motor controller board through XBee wireless. The motor controller board receives the weight information and sends necessary information to the motor driver what speed the motor should be set to. Then the energy meter measures how much energy is being used by the motor. The motor microcontroller collects information about the speed, timing and power for the customer. The customer then able to access the information collected through a SD Card that stores the information from the motor controller board. With this data the customer can compare energy consumption and traveled time under two different policies which are variable and constant speed.

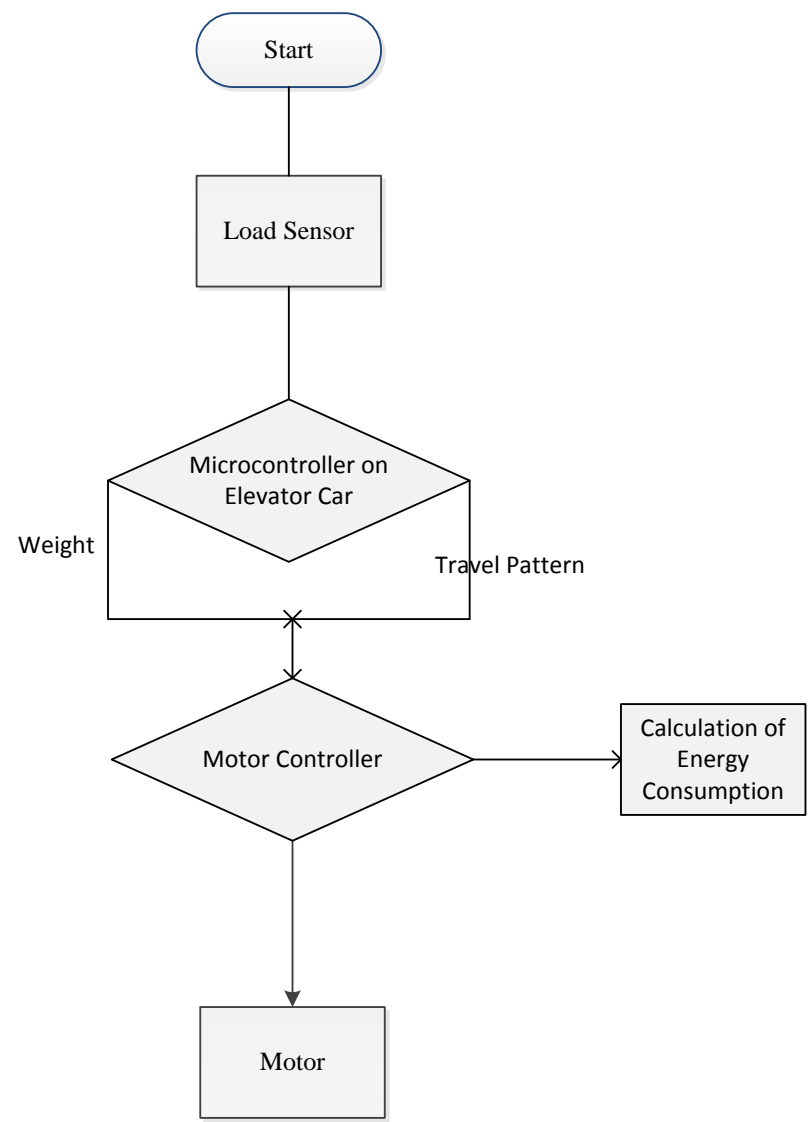

Figure 4. Block diagram of the design of energy efficient elevator conservation system

The Sensor board controller communicates with the load sensor and sends information about the load on the motor via XBEE Pro Module to the Motor Control Board. From left to right we begin with the Sensor Interface Block which powers the sensor and also sends an analog output from $0-20 \mathrm{mV}$ into the Amplification Block. This is a non-inverting OP AMP circuitry block designed to amplify the sensors small change in millivolts to a more repeatable range of $0-3 \mathrm{~V}$. The signal will be passed through a band pass filter in order to clear noise and extract the signal containing information. This analog signal will then be converted by an 8 bit Analog to Digital converter that is onboard a microcontroller. The microcontroller is responsible for sending the information about load on the shaft of the motor as well as the traffic pattern extracted from the SD/USB interface. The micro controller uses serial peripherals to communicate with the SD/USB and a UART communication with the XBEE Pro module. The Power supply for this PCB is 12 volt wall wart that will have DC to DC conversions from 12 volts to 3.3 volts and 10 volts of excitation for the sensor. 
It is found from the experiment that, after using this controller to control the motor of the traction elevator, it minimizes the consumed energy of $3 \mathrm{KJ}$ and reduces the travel time of around $26 \mathrm{sec}$ of a traditional traction elevator system. These results indicate that, this microcontroller based conservation system is very much efficient to control the elevator system for reducing energy consumption and enhancing the performance.

\section{CONCLUSION}

This paper presents a design principle of elevator conservation systems which is able to minimize the energy consumption of existing traction elevator system. By using microcontroller as a controlling device, this work aims to control the elevator and enhance the performance. This controller is able to control the elevator system in different modes like fixed speed or variable speed or variable frequency and can able to decrease travel time. As microcontroller is being used here as a controller, it is very much chip and easy to control and can be made without any dependency to external hardware.

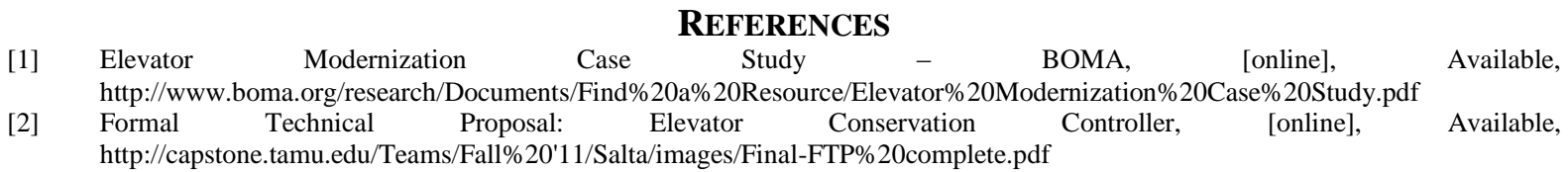

[3] Department Of Measurement and Information Systems, Microprocessor based elevator controlling system, version: FLV-V02.1, 1989 (Project report in Hungarian).

[4] Microprocessors and Interfacing (Programming \& Hardware)-Douglas V.Hall.

[5] Poorvi Behre, Viveka Nema, and Bhupendra Badoniya, Congestion-Free Elevator Control Using Microcontroller, International Journal of Scientific \& Engineering Research Volume 4, Issue 1, January-2013 1, ISSN 2229-5518.

[6] R.K. PATJOSHI, Design and implementation of embedded based elevator control system, National Institute of Technology, Rourkela, MT, 2010. 